\title{
Assistive Devices for Patients with Auditory Neuropathy: Hearing Aid Use
}

\author{
Rosamaria Santarelli, M.D., ${ }^{1}$ Roberta Rossi, M.D., ${ }^{1}$ and \\ Edoardo Arslan, M.D. ${ }^{1}$
}

ABSTRACT

${ }^{1}$ Department of Neuroscience, Service of Audiology and Phoniatrics, University of Padova, Padova, Italy.

Address for correspondence and reprint requests: Rosamaria Santarelli, M.D., Department of Neuroscience, Service of Audiology and Phoniatrics, University of Padova, Via Giustiniani 2, I-35128 Padova, Italy (e-mail: rosamaria. santarelli@unipd.it).

Auditory neuropathy (AN) is a hearing disorder characterized by disruption of temporal coding of acoustic signals in the auditory nerve resulting from lesions involving auditory nerve fibers, the inner hair cells, or their synapses with auditory nerve terminals. Disruption of auditory nerve discharge underlies both the absence of auditory brainstem responses (ABRs) and impairment of speech perception. AN may be related to genetic disorders or result from a wide range of other etiologies. It can be identified either as an isolated disorder (isolated AN) or associated with multisystem involvement (non-isolated AN). Effectiveness and choice of assistive devices depend crucially upon etiology, site of lesion, and stage of the disease. Cochlear implants constitute an effective rehabilitative tool able to restore speech perception in many patients with genetic AN, especially those affected by the isolated form of the disorder. Some children with isolated AN have proved to be good hearing aid users, showing satisfactory open-set speech perception abilities in the aided condition. Over $50 \%$ of children discharged from neonatal intensive care units showing the electrophysiological profile of AN (absent ABRs, presence of otoacoustic emissions) benefit from hearing aid use, the provision of amplification resulting in remarkable improvement in speech perception. It can be concluded that differences in cochlear implant outcome or hearing aid use in patients with AN are related to the pathophysiological mechanisms underlying alteration of auditory nerve discharge associated with individual etiologies.

KEYWORDS: auditory neuropathy, hearing aids, cochlear implants, otoferlin, prematurity 
Learning Outcomes: As a result of this activity, the participant will be able to (1) list the main mechanisms underlying the alteration of auditory nerve discharge in auditory neuropathy, and (2) compare the benefits of hearing aid and cochlear implant use for patients with auditory neuropathy.

\section{DEFINITION AND CLASSIFICATION OF AUDITORY NEUROPATHY}

Auditory neuropathy (AN) is a hearing disorder characterized by disruption of temporal coding of acoustic signals in auditory nerve fibers resulting in impairment of auditory perceptions relying on temporal cues. ${ }^{1,2}$ Abnormal discharge of auditory fibers results from lesions involving the nerve fibers themselves (postsynaptic AN) or the inner hair cells (IHCs) and their synapses with auditory nerve terminals (presynaptic AN). ${ }^{1}$ The disruption of auditory nerve discharge underlies both the absence, or profound alterations, of auditory brainstem responses (ABRs) and severe impairment of speech perception. In contrast, cochlear receptor outer hair cell $(\mathrm{OHC})$ activities are preserved. This is indicated by detection of otoacoustic emissions (OAEs) and by recording of the cochlear microphonic $(\mathrm{CM}){ }^{1,3}$

AN may be congenital or acquired., ${ }^{4,5}$ Congenital AN affects language development strictly related to the existence of a sensitive period that declines with age. ${ }^{6}$ In this period, the development of language skills is strictly dependent on the cortical plasticity processes and requires an efficient auditory input to be accomplished. ${ }^{6}$ When the onset of $\mathrm{AN}$ is delayed to childhood or adolescence (acquired AN), abnormalities of auditory input lead to severe impairment of speech perception and eventually progressive deterioration of acquired language skills. ${ }^{1}$

Both congenital and acquired forms of AN may be underlain by genetic disorders or result from a wide range of other etiologies (infectious, toxic-metabolic, immunologic). ${ }^{4,5} \mathrm{Nev}-$ ertheless, no etiologic factors can be identified in about half of patients. ${ }^{1}$ All forms of the disorder may be present in isolation (isolated AN) or associated with multisystem involvement including peripheral and/or optic neuropathies (non-isolated AN). ${ }^{5}$ Table 1 reports the genetic disorders that have so far been associated with AN. ${ }^{5}$ Genes and loci implicated in the pathogenesis of the disorder with the corre- sponding phenotypes are indicated. Both presynaptic and postsynaptic mechanisms have been hypothesized for isolated forms whereas non-isolated AN is always considered as underlain by postsynaptic lesion. ${ }^{5}$

AN occurs in all age groups and reported prevalence varies from 1 to $10 \% .^{1}$ The high degree of variability may reflect the inclusion in some studies of patients showing the electrophysiological profile of "absent ABR and presence of OAEs," which is not specific to AN. For instance, in some neonates discharged from neonatal intensive care units (NICUs) who show absent $\mathrm{ABR}$ and presence of OAEs at newborn hearing screening, ABR abnormalities may reflect delayed maturation of both brainstem and auditory nerve generators. ${ }^{5}$ On the other hand, some hearing disorders underlying AN might not have been included in prevalence estimates because identification of the $\mathrm{AN}$ picture may require several further tests to be performed in addition to hearing threshold estimation and these are not invariably included in routine diagnosis.

Clinical criteria for diagnosis include impairment of speech perception beyond that expected for the hearing loss, absence or marked abnormality of ABRs, and preservation of $\mathrm{OHC}$ activities (OAEs and/or CM). ${ }^{3,5}$ Nevertheless, some patients with AN may show normal hearing thresholds ${ }^{7}$ and impairment of speech perception is apparent only in the presence of noise. ${ }^{2}$ In these subjects the evaluation of speech perception in noise and psychoacoustical testing (gap detection, frequency discrimination) are mandatory.

Decrease in auditory input and alterations in temporal coding of acoustic signals underlie both ABR abnormalities and impairment of speech perception. Both presynaptic $A N$ and postsynaptic AN lead to reduced auditory input, impairment of spike initiation, and slowing in conduction velocity in auditory fibers, all resulting in disruption of auditory nerve discharge. No information regarding cochlear nerve and hair cell activities are available 
Table 1 Genes and Loci Implicated in the Pathogenesis of AN with Corresponding Phenotypes

\begin{tabular}{|c|c|c|c|c|c|}
\hline & Locus & Gene & Transmission & Phenotype & Reference \\
\hline \multicolumn{6}{|l|}{ Isolated AN } \\
\hline & $2 p 23-p 22$ & OTOF & Recessive & $\begin{array}{l}\text { Congenital profound } \\
\text { deafness }\end{array}$ & Varga, $2003^{35}$ \\
\hline & $2 q 31.1-q 31.3$ & PJVK & Recessive & $\begin{array}{l}\text { Congenital profound } \\
\text { deafness }\end{array}$ & Delmaghani, $2006^{36}$ \\
\hline & $\begin{array}{l}13 q 21-q 24 \\
m t D N A\end{array}$ & $\begin{array}{l}\text { DIAPH3 } \\
12 S \text { rRNA }\end{array}$ & Dominant & Moderate deafness & $\begin{array}{l}\text { Schoen, } 2010^{37} \\
\text { Wang, } 2005^{38}\end{array}$ \\
\hline \multicolumn{6}{|l|}{ Non-isolated } \\
\hline \multicolumn{6}{|l|}{ AN } \\
\hline$\overline{\mathrm{CMT}} 1 \mathrm{~A}$ & 17p11.2-p12 & PMP22 & Dominant & $\begin{array}{l}\text { Mild/severe deafness; } \\
\text { demyelinating } \\
\text { neuropathy }\end{array}$ & Kovach, $2002^{39}$ \\
\hline CMT 1B & $1 q 22$ & $M P Z$ & Dominant & $\begin{array}{l}\text { Mild/severe deafness; } \\
\text { demyelinating } \\
\text { neuropathy }\end{array}$ & Starr, $2003^{12}$ \\
\hline CMT 2E & $8 p 21$ & $N F-L$ & Dominant & $\begin{array}{l}\text { Normal hearing; } \\
\text { axonal neuropathy }\end{array}$ & Butinar, $2008^{14}$ \\
\hline CMT 4D & $8 q 24.3$ & NDRG 1 & Recessive & $\begin{array}{l}\text { Mild/severe } \\
\text { deafness; axonal/ } \\
\text { demyelinating } \\
\text { neuropathy }\end{array}$ & Kalaydijeva, $2000^{40}$ \\
\hline $\mathrm{CMT}$ & 1 p34 & GJB3 (Cx31) & Dominant & Mild deafness & Lopez-Bigas, $2001^{41}$ \\
\hline CMT 1X & Xp13 & GJB1 (CX32) & $\begin{array}{l}\text { X-linked, } \\
\text { dominant }\end{array}$ & $\begin{array}{l}\text { Demyelinating } \\
\text { neuropathy }\end{array}$ & Bahr, $1999^{42}$ \\
\hline ADOA & $3 q 28-q 29$ & OPA1 (R445H) & Dominant & $\begin{array}{l}\text { Optic neuropathy; } \\
\text { moderate deafness }\end{array}$ & $\begin{array}{l}\text { Amati-Bonneau, } \\
2005^{43}\end{array}$ \\
\hline AROA & $11 q 14.1-11 q 22.3$ & TMEM126A & Recessive & $\begin{array}{l}\text { Optic neuropathy; } \\
\text { mild hearing loss }\end{array}$ & Meyer, $2010^{44}$ \\
\hline Friedreich & $9 q 13$ & $F X N$ & Recessive & $\begin{array}{l}\text { Ataxia; axonal neuropa- } \\
\text { thy; optic neuropathy; } \\
\text { cardiomyopathy; normal } \\
\text { hearing threshold/mild } \\
\text { deafness }\end{array}$ & Rance et al, $2008^{45}$ \\
\hline AUNX1 & $X q 23-q 27.3$ & & $\begin{array}{l}\text { X-linked, } \\
\text { recessive }\end{array}$ & $\begin{array}{l}\text { Sensory axonal } \\
\text { neuropathy; mild/ } \\
\text { severe deafness }\end{array}$ & Wang et al, $2006^{46}$ \\
\hline $\begin{array}{l}\text { DDON } \\
\text { (Mohr-Traneb.) }\end{array}$ & $X q 22.1$ & TIMM8A & $\begin{array}{l}\text { X-linked, } \\
\text { recessive }\end{array}$ & $\begin{array}{l}\text { Progressive deafness; } \\
\text { dystonia, optic neuropa- } \\
\text { thy; dementia }\end{array}$ & Bahmad, $2007^{47}$ \\
\hline $\begin{array}{l}\text { LHON } \\
\text { (Leber) }\end{array}$ & mtDNA & MTDN4 & & $\begin{array}{l}\text { Optic neuropathy; } \\
\text { mild/moderate } \\
\text { deafness }\end{array}$ & $\begin{array}{l}\text { Ceranic and } \\
\text { Luxon, } 2004^{48}\end{array}$ \\
\hline
\end{tabular}

Abbreviation: AN, auditory neuropathy.

through $\mathrm{ABR}$ recordings due to the low signalto-noise ratio. Abnormal discharge of auditory nerve fibers may be identified through transtympanic electrocochleography (ECochG) re- cording, which has been proposed to define the features of both receptor (summating potential [SP], CM) and auditory nerve potentials (compound action potential $[\mathrm{CAP}])$ in $\mathrm{AN} .^{8}$ 


\section{Summating Potential (SP) Compound Action Potential (CAP)}
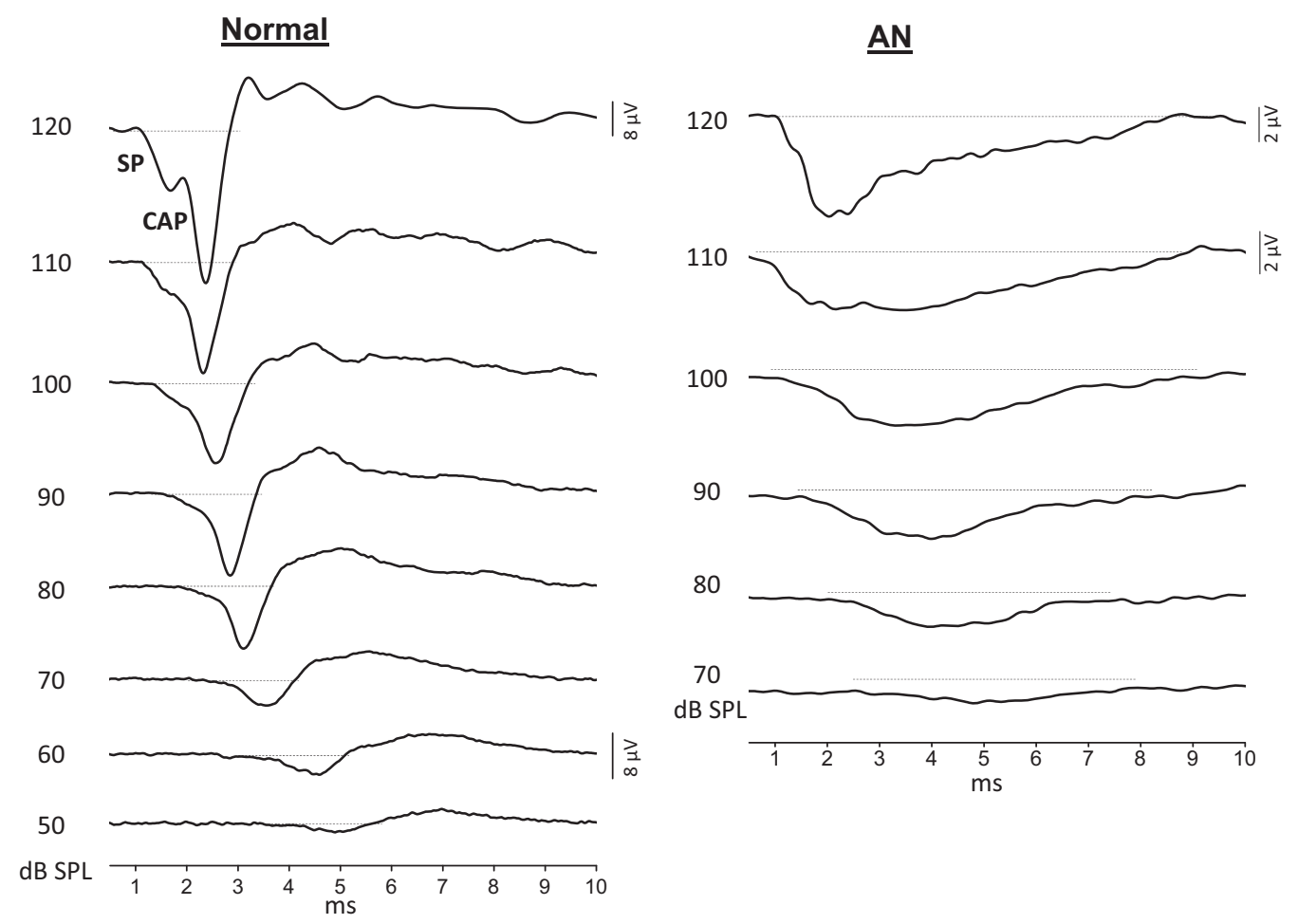

Figure 1 Electrocochleography (ECochG) potentials obtained from one normally hearing child (left side) and one patient with auditory neuropathy (AN) (right side). The responses collected from the normally hearing ear show the compound action potential (CAP) at decreasing stimulation intensities from 120 to $60 \mathrm{~dB}$ peak equivalent sound pressure level (p.e. SPL); the maximum stimulus intensity used in our laboratory is $120 \mathrm{~dB}$ p.e. SPL corresponding to $90 \mathrm{~dB}$ normalized Hearing Level ( $\mathrm{nHL}$ ). The summating potential (SP) is identifiable as a rapid negative potential preceding the CAP at intensities from 120 to $80 \mathrm{~dB}$ p.e. SPL. The most common pattern of ECochG response observed in patients with AN consists of a prolonged low-amplitude negative deflection showing no separation between SP and CAP. Note the decrease in amplitude and the broadening in duration of the negative potential recorded from the subject with AN compared to the CAP obtained from the normal ear.

CMs are recorded from patients with AN with normal or enhanced amplitude. ${ }^{8}$ This finding supports the hypothesis of normal functioning of $\mathrm{OHCs}$, at least at an early stage of the disease. After CM cancellation, the ECoch $\mathrm{G}$ response recorded from the majority of patients with AN consists of a prolonged low-amplitude negative potential showing no separation between SP and CAP. An example is reported in Fig. 1, which shows the ECochG recordings obtained at various stimulation intensities from one patient with AN underlain by Charcot-Marie-Tooth disease together with the responses recorded from one normally hearing ear. The ECochG response found in the normally hearing ear, which consists of a rapid negative $\mathrm{SP}$ followed by a straight $\mathrm{CAP}$, is replaced in the patient with AN by a lowamplitude negative wave that appears remarkably prolonged in duration.

The prolonged negative potentials recorded from patients with AN result from reduced auditory input and dispersed activation of auditory nerve fibers. ${ }^{8}$ According to identification of a small CAP component superimposed on the prolonged activity at highstimulation intensity and to effects of adaptation induced by high-rate stimuli, three patterns of ECochG responses have been recognized in patients with $\mathrm{AN}$ possibly related 
to different mechanisms and sites of lesion. ${ }^{8}$ These findings may help to shed light on the pathophysiological mechanisms underlying the alteration of auditory nerve discharge in AN. Moreover, the identification of abnormal ECoch $G$ patterns may provide crucial information in patients for whom the diagnosis of $\mathrm{AN}$ is not straightforward. Indeed, OAEs are absent in over one-third of patients on retesting, ${ }^{1}$ whereas speech perception as evaluated in quiet environments has been reported to be comparable to that of normally hearing listeners in some AN subjects. ${ }^{7}$

\section{ETIOLOGY AND}

\section{PATHOPHYSIOLOGICAL}

\section{MECHANISMS: IMPLICATIONS FOR} USE OF ASSISTIVE DEVICES

In the healthy cochlea, temporal precision of acoustic signaling is guaranteed by the fast kinetics of synaptic release, postsynaptic membrane activation, and initiation and propagation of spikes along the auditory nerve fibers. Lesions interfering with the fast dynamics of acoustic information processing at any level from the cochlea to the auditory nerve may disrupt temporal coding in the auditory nerve fibers. Thus, abnormal nerve fiber discharge may result from different mechanisms and these, in turn, are related to different etiologies. One typical example of presynaptic disorder leading to the clinical picture of $\mathrm{AN}$ is the abnormal function of otoferlin. Reduced activity of this protein leads to reduced vesicle replenishment ${ }^{9}$ and elimination of the fast phase of exocytosis. ${ }^{10}$ Prolonged neural potentials have been recorded by transtympanic ECochG from children with mutations in the OTOF gene. ${ }^{11}$ These have been interpreted as resulting from abnormal postsynaptic activation due to both reduction of neurotransmitter availability at the synaptic cleft and impairment of multivesicular release with generation of small excitatory postsynaptic potentials (EPSPs) with abnormal morphology and dispersed in time. ${ }^{11}$ These mechanisms are relevant from the point of view of rehabilitation programs because cochlear implants are likely to constitute the only useful tool for restoring auditory perception in children with mutations in the OTOF gene through electrical stimulation of auditory nerve fibers, which are believed not to be directly involved by the pathological process.

Postsynaptic membrane and auditory nerve fibers also are suited for fast and precise signal transmission in the healthy cochlea. Demyelination of auditory nerve fibers, which is observed in demyelinating neuropathies, is expected to result in slowed conduction velocity with consequent disruption of temporal coding of acoustic signals. ${ }^{2}$ Moreover, the decrease in number of auditory fibers found in axonal neuropathies would result in reduced auditory input to the brainstem. ${ }^{1}$ Indeed both reduction in number of auditory nerve fibers and demyelination of spared axons have been observed on postmortem examination in the auditory nerve of patients affected by Charcot-Marie-Tooth disease. ${ }^{12}$ It is reasonable to expect that the outcome of cochlear implantation in this category of patients would depend critically on the number of spared axons and the extension of their involvement, whereas the effectiveness of acoustic amplification would be completely impeded by the desynchronization of the acoustic information in the auditory nerve due to demyelination.

Another point to be considered in postsynaptic disorders concerns the site and extension of lesions. For instance, combined electrophysiological and audiological evaluation obtained from two related patients carrying the $\mathrm{R} 445 \mathrm{H}$ mutation in the OPA1 gene has suggested that in this disorder the lesion involves the distal portion of auditory nerve fibers. ${ }^{13}$ This hypothesis is supported by a mouse model of OPA1 showing dendritic pruning of the optic nerve fibers at an early stage of the disease. ${ }^{14}$ These findings are relevant from the point of view of the outcome of cochlear implantation, because electrical stimulation through the cochlear implant has restored speech perception in some patients with the mutation in the OPA1 gene, possibly by bypassing the lesion site with consequent stimulation of the preserved portion of auditory nerve fibers.

Extensive phenomena of demyelination involving the whole auditory nerve have been found on postmortem examination in one patient with advanced OPA1 disease. ${ }^{15}$ This 
finding, together with the loss of retinal ganglion cells and degeneration of residual axons found in one mouse model ${ }^{16}$ would suggest that demyelination and axonal loss affecting the whole nerve constitute a more advanced stage of the disease compared to the stage of dendritic tree pruning. The outcome of cochlear implantation is expected to be poor in these cases of extensive involvement of auditory nerve with little or no preservation of functioning nerve fibers.

Several nongenetic etiologies are associated with AN. Among them, the electrophysiological profile of AN (absent ABRs and presence of OAEs) has been found in several children discharged from NICUs. ${ }^{17,18}$ This finding has attracted a great deal of attention recently in an effort to improve newborn hearing screening procedures and to refine the diagnosis of hearing impairment. The analysis of possible mechanisms underlying the electrophysiological profile of $\mathrm{AN}$ in these children deserves consideration. First of all, in addition to an increased hearing threshold, ABR absence may result from reduced synchrony occurring at any level from auditory nerve to brainstem generators. A reduced correlation between ABR and hearing thresholds has been documented in premature babies and in those suffering perinatal asphyxia ${ }^{19-21}$ as well as in children showing neurological diseases involving the central nervous system (CNS) ${ }^{22}$ In these cases, alterations of ABRs might ensue from abnormal firing of brainstem generators, which retain little or no connection with the dynamics of auditory periphery activation. In addition, the combined effects of prematurity and other risk factors acting in NICUs, chiefly among them perinatal asphyxia, impinge on cochlear function, resulting in various types and degrees of damage. ${ }^{17}$ Postmortem examination performed on temporal bones of deceased neonates has shown extensive hair cell loss with a higher frequency of selective IHC loss in premature infants compared to full-term babies. ${ }^{23}$ On the basis of these findings, the dysfunction of IHCs has been proposed as the primary mechanism underlying nongenetic forms of $\mathrm{AN}$, at least in children discharged from NICUs. ${ }^{23}$ In this view, an increase of the acoustic input as obtained through amplification with hearing aids could possibly compensate for the loss of hair cells, ${ }^{1}$ the effectiveness depending on the total number of residual receptor elements and the proportion of spared OHCs and IHCs.

In conclusion, the choice and effectiveness of assistive devices proposed for patients with AN depend critically on the mechanisms underlying the alteration of auditory nerve firing and thus, on the etiology of AN disorders. In this view, the evaluation of assistive device benefit only can be obtained through "well-controlled, longitudinal prospective studies that provide homogeneous grouping of participants."24(168)

\section{REHABILITATIVE OPTIONS}

Both hearing aids and cochlear implants have been proposed for rehabilitation of patients with AN. ${ }^{1,13,24-26}$ Nevertheless, the heterogeneity of the disorder, the incomplete knowledge of the pathological alterations and mechanisms underlying individual diseases, and the evaluation of the outcome, performed in heterogeneous groups of subjects, all make it difficult to draw any conclusions.

Cochlear implantation is considered the treatment of choice for restoring speech perception in patients with AN. ${ }^{1}$ However, the outcome of cochlear implantation varies widely among patients. Although it is generally acknowledged that subjects with presynaptic AN due to mutations in the OTOF gene invariably benefit from cochlear implant use, ${ }^{1,27}$ poor outcome has been reported for patients presumed to be affected by postsynaptic disorders possibly involving the entire auditory nerve such as Friedreich's ataxia and deafness-dystonia-optic neuropathy (DDON) syndrome. ${ }^{28,29}$ However, two patients showing the $\mathrm{R} 445 \mathrm{H}$ mutation in the OPA1 gene and some patients with mutation in the DIAPH3 gene have shown restoration of both speech perception and ABRs following cochlear implantation. ${ }^{13,30}$ The good outcome of cochlear implantation in these cases has been explained by hypothesizing that the lesion was confined to the distal portion of auditory nerve fibers, at least in the early stage of the disease. ${ }^{13,30}$

Several studies including large numbers of children affected by congenital AN have 
reported that many of them benefit from cochlear implant use..$^{25,26,31}$ It is important to outline that a significant proportion of the children included in these studies have been discharged from NICUs. They are believed then to share the same pathological alterations at the level of the auditory periphery presumably consisting of predominant involvement of IHCs with preservation of auditory nerve fibers. $^{23,32}$

Hearing aids have always been considered of extremely limited use in AN. Indeed, acoustic amplification seems to be ineffective in acquired $\mathrm{AN}$ as the majority of patients refuse to wear hearing aids. In contrast, several studies have reported that children with congenital AN may benefit from hearing aid use. Rance and colleagues reported that over $50 \%$ of children affected by congenital forms of AN are good hearing aid users, the provision of amplification resulting in significant improvement of openset speech recognition scores. ${ }^{32}$ Specifically, hearing aid users achieved a mean $67 \%$ score on Phonetically Balanced Kindergarten (PB-K) word testing with a mean difference between unaided and aided conditions of 57\%. Moreover, Teagle and colleagues, who performed a prospective study including 140 children with congenital AN who had been identified through a newborn hearing screening program, reported that the percentage of hearing aid users was $31 \% .{ }^{26}$ Interestingly, the majority of children included in both studies had been discharged from NICUs. It can be hypothesized that in children with congenital AN admitted to the NICU, an increase of signal intensity through acoustic amplification might compensate for the decreased input resulting from loss of IHCs through recruitment of residual receptor elements in the absence of auditory nerve involvement. ${ }^{1,23}$

The majority of children included in the studies previously reported had a difficult neonatal course, which could result in CNS dysfunction in a high proportion of infants. The coexistence of CNS involvement makes it quite challenging if not impossible to evaluate speech perception abilities and also presumably impinges on language development. ${ }^{25,26}$ Despite these limitations, a remarkable proportion of these children did benefit from hearing aid use.
Considering the potential benefit of hearing aid use in patients with $\mathrm{AN}$, the Joint Committee on Infant Hearing (2007) recommended that children diagnosed with AN undergo a trial with acoustic amplification before cochlear implantation. ${ }^{17}$ According to Rance and colleagues, only the children showing scores lower than $35 \%$ on the PB-K word test performed in the aided condition should be selected for cochlear implantation independently of their pure tone sensitivity. ${ }^{32}$ Thus, cochlear implantation constitutes the treatment of choice for all children with AN with profound deafness regardless of the underlying etiology due to the invariably poor speech perception abilities found in the aided condition. In contrast, wide differences in speech perception abilities have been observed among children with AN showing moderate hearing loss. In these cases, the decision for cochlear implantation should be delayed to obtain an evaluation of speech perception abilities and language development over time. ${ }^{32}$

In conclusion, differences in the effectiveness of assistive device use in patients with AN are deemed to arise from different sites of lesion and pathophysiological mechanisms underlying. both acquired and congenital forms. Bearing in mind the reported findings and comments, the issue of hearing aid use in $\mathrm{AN}$ will be addressed in the following sections by tentatively grouping patients by etiology, suggested pathophysiological mechanism, and site of lesion.

\section{ISOLATED AN}

Patients with AN diagnosed at the University of Padua Service of Audiology and Phoniatrics are classified into several groups in Table 2 according to etiology and the association of multisystem involvement.

Among the isolated forms, biallelic mutations in the OTOF gene result in congenital profound hearing loss in the majority of patients. One example is reported in Fig. 2, showing the absence of ABRs associated with the detection of distortion product otoacoustic emissions. ECochG recordings show the lowamplitude prolonged negative response that is identified at very low stimulation intensities despite profound hearing loss. Notice the 
Table 2 Cochlear Implant and Hearing Aid Users

\begin{tabular}{|c|c|c|c|c|c|}
\hline & Etiology & Users & $\begin{array}{l}\text { Cochlear } \\
\text { Implant }\end{array}$ & $\begin{array}{l}\text { Hearing Aids } \\
\text { before } \\
\text { Cochlear } \\
\text { Implant }\end{array}$ & $\begin{array}{l}\text { Hearing } \\
\text { Aids }\end{array}$ \\
\hline \multicolumn{6}{|c|}{ Isolated AN } \\
\hline & OTOF & $8 / 9$ & 8 & 6 & \\
\hline & $\mathrm{C} \times 26$ & $1 / 1$ & & & 1 \\
\hline & Familiarity & $6 / 10$ & 5 & 2 & 1 \\
\hline & Unknown & $13 / 14$ & 10 & 5 & 3 \\
\hline \multicolumn{6}{|c|}{ Non-isolated AN } \\
\hline & OPA1 & $7 / 9$ & 7 & 2 & \\
\hline & Scleroderma & 1 & 1 & 1 & \\
\hline & Kasabach-Merritt & 1 & 1 & & \\
\hline & Unknown & $1 / 3$ & 1 & & \\
\hline $\mathrm{NICU}$ & & $17 / 23$ & 4 & 3 & 13 \\
\hline
\end{tabular}

Cochlear implant and hearing aid users among patients with AN diagnosed at the University of Padua Service of Audiology and Phoniatrics. Patients have been classified according to etiology and the association of multisystem involvement. The "Users" column reports the number of subjects using assistive devices expressed as a ratio of total number of patients diagnosed with the same disorder. Columns "Cochlear implant" and "Hearing Aids before Cochlear Implant" refer respectively to patients submitted to cochlear implantation and to those who had used hearing aids before undergoing surgery. The "Hearing Aid" column reports the number of subjects who only use hearing aids due to a good outcome with acoustic amplification or to refusing cochlear implantation. Abbreviations: AN, auditory neuropathy; NICU, neonatal intensive care unit.

presence of a small CAP superimposed on the prolonged activity at high-stimulus intensity.

Of the nine patients with biallelic mutations in the OTOF gene followed up at our institution, eight underwent cochlear implantation by 2 years of age. Disyllabic word openset recognition reached an 80 to $90 \%$ correct score by 1 year of cochlear implant use. All children had been fitted with power hearing aids prior to cochlear implantation and showed reasonable functional gain from 0.25 to $1 \mathrm{kHz}$ (Fig. 2). ${ }^{34}$ Nevertheless, speech perception scores as evaluated with hearing aid use on disyllabic word recognition were lower than $30 \%$ in the aided condition for all children. Therefore, acoustic amplification provided patients with an auditory input that resulted in substantial improvement of hearing sensitivity and development of some speech perception prior to cochlear implantation. The improvement of pure tone sensitivity can be explained by hypothesizing that increasing acoustic input results in a large amount of neurotransmitter release with consequent enhancement of postsynaptic membrane activation and spike triggering. This hypothesis is corroborated by the identification of a small CAP at high-stimulus intensity in ECochG recordings (Fig. 2).

With regard to other patients showing isolated AN (Table 2), one carried biallelic mutation in the GJB2 gene; in six subjects the disorder was familiar although no gene mutation was identified, whereas in the remaining subjects, no etiologic factors were found. Fifteen of 24 subjects (nine children and six adults) underwent cochlear implantation with a good outcome as the percentage of correct scores on open-set disyllabic word recognition varied between 70 and $100 \%$ by 1 year of cochlear implant use. Of these, seven patients had worn hearing aids before cochlear implantation with improvement of hearing sensitivity; however, mean scores on open-set disyllabic word recognition were lower than $10 \%$ in the aided condition. Therefore, also for patients with isolated AN not related to mutations in the OTOF gene, using acoustic amplification improved hearing sensitivity but not speech perception.

Three patients with isolated AN were "pure" hearing aid users. Among them, one girl showing profound hearing loss due to 


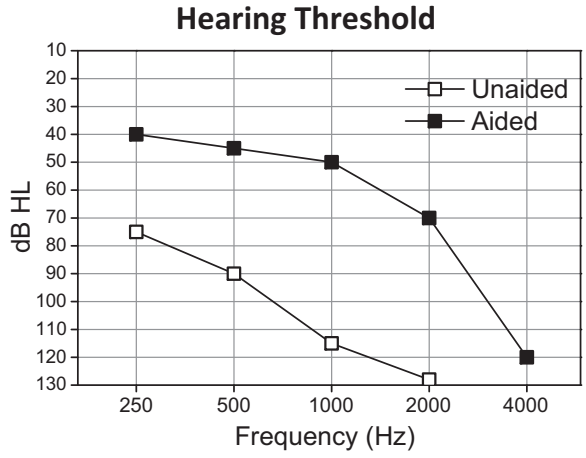

DPOAEs
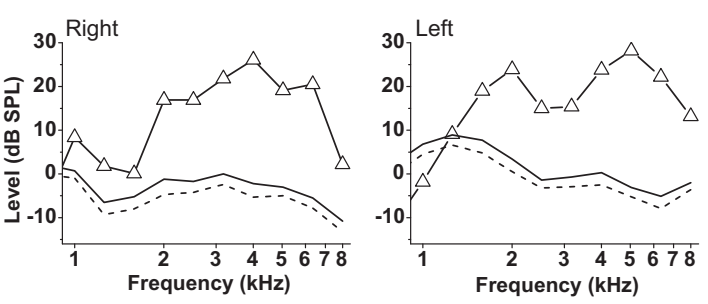

Auditory Brainstem Responses (ABRs)

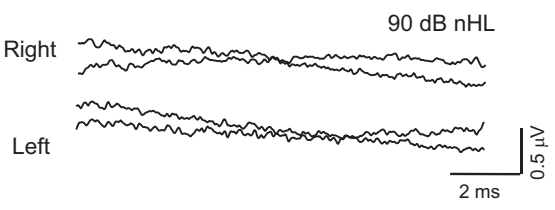

\section{Electrocochleography (ECochG)}

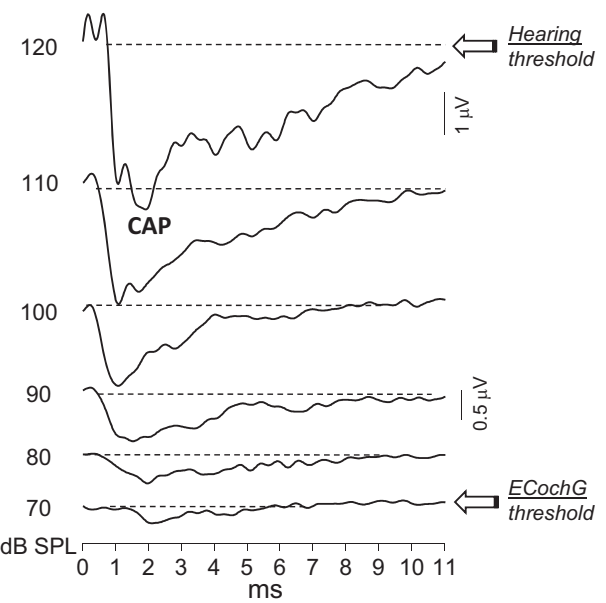

Figure 2 Hearing thresholds, distortion product otoacoustic emissions (DPOAEs), auditory brainstem responses (ABRs), and electrocochleography (ECochG) potentials collected from one child with biallelic mutation in the OTOF gene. Audiometric evaluation (upper left) was performed in the free-field by visual reinforced audiometry. Both unaided and aided thresholds, which were obtained with the child wearing both hearing aids, are reported. Notice that the functional gain was good, but the aided thresholds at high frequencies appeared beyond the intensity range calculated for conversational speech. ${ }^{34}$ DPOAEs were recorded from both ears whereas ABRs were absent. ECochG recordings showed the presence of the typical prolonged negative potential, which was recorded as low as $70 \mathrm{~dB}$ sound pressure level (corresponding to 40 $\mathrm{dB}$ normalized Hearing Level $(\mathrm{nHL}))$ despite the profound hearing loss estimated on the basis of audiometric evaluation.

biallelic mutation in the GJB2 gene used acoustic amplification because the parents refused cochlear implantation. Interestingly, one child affected by congenital AN of unknown etiology did benefit from hearing aid use (Fig. 3). He had passed neonatal hearing screening with OAEs and was diagnosed with severe hearing loss at the age of 18 months on the basis of parental concern about sound sensitivity. ABRs were absent and ECochG recordings showed the typical pattern of prolonged negative potentials. Hearing threshold evaluation obtained by visual reinforced audiometry indicated severe hearing loss. The child was fitted with power hearing aids and the aided thresholds showed good functional gain from 0.25 to $4 \mathrm{kHz}$. He reached a $100 \%$ correct score on both open-set disyllabic word and sentence recognition with hearing aid use as evaluated at the age of 3 years. Because the development of expressive language also showed remarkable progress over time, this child has not been selected for cochlear implantation thus far.

The third subject was a 14-year-old girl showing isolated AN with unknown etiology initiated in childhood, with difficulty in understanding speech. Audiometry indicated moderate hearing loss; OAEs were recorded from both ears and ABRs were absent. Also in this case, prolonged negative potentials were recorded by transtympanic ECochG. The girl was fitted with hearing aids and showed reasonable speech perception performance with the use of acoustic amplification as correct scores on open-set recognition of disyllabic words and sentences were 65 and 95\%, 
DPOAEs

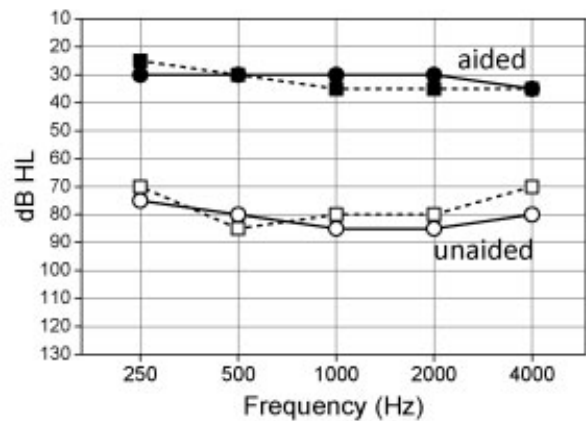

DPOAES
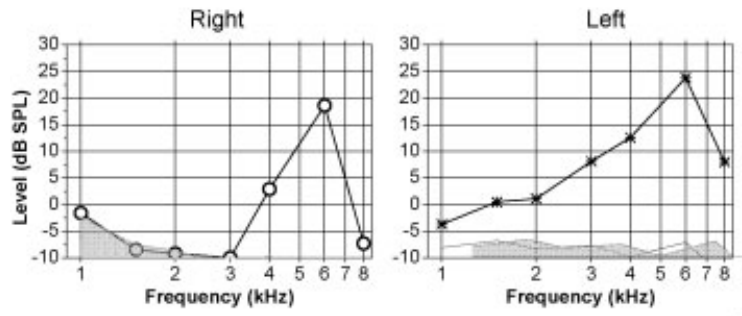

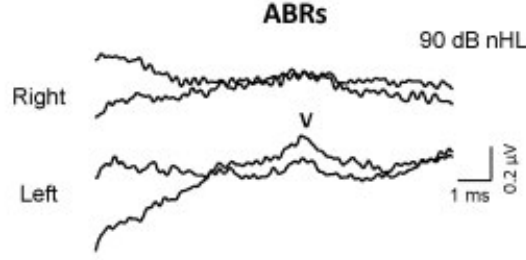

Electrocochleography (ECochG)

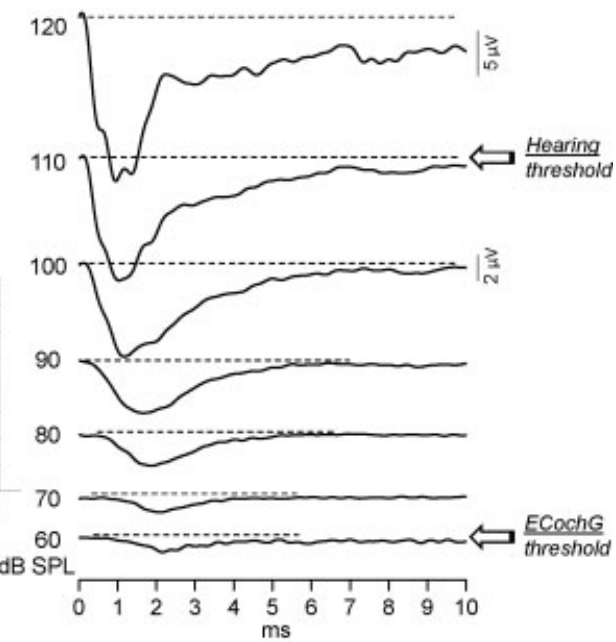

Figure 3 Hearing thresholds, distortion product otoacoustic emissions (DPOAEs), auditory brainstem responses (ABRs), and electrocochleography (ECochG) potentials collected from one child with isolated auditory neuropathy (AN). Audiometric evaluation was performed by visual reinforced audiometry. Unaided thresholds were obtained with headphones whereas aided thresholds were evaluated separately for each ear. Notice that the aided thresholds appeared within the intensity range calculated for conversational speech at all the tested frequencies. ${ }^{34}$ DPOAEs were detected in both ears whereas ABRs were absent. Also in this child the ECochG recordings showed the presence of the typical prolonged negative potential, which was recorded as low as $60 \mathrm{~dB}$ peak equivalent sound pressure level (corresponding to $30 \mathrm{~dB}$ normalized Hearing Level $(\mathrm{nHL}))$ despite the profound hearing loss estimated on the basis of audiometric evaluation.

respectively. She also used lipreading for communication and was able to use the speakerphone. This patient was not selected for cochlear implantation due to the reasonably good speech perception abilities.

In conclusion, a trial with hearing aids in patients with isolated AN is highly recommended before considering the option of $\mathrm{co}^{-}$ chlear implant use. Indeed, acoustic amplification provides the majority of patients with an acoustic input prior to cochlear implantation. This may help to prevent colonization of secondary auditory cortical areas by other sensory modalities, particularly visual information. $^{33}$ Importantly, some patients with isolated AN may turn out to be good hearing aid users.

\section{NON-ISOLATED AN}

The majority of subjects with non-isolated AN followed up at our institution (Table 2) underwent cochlear implantation. The outcome was more variable compared with that found in isolated $\mathrm{AN}$ as two patients out of eight scored less than $30 \%$ on open-set disyllabic word recognition by 2 to 3 years of cochlear implant use. In the remaining subjects, correct scores on a disyllabic recognition test ranged from 50 to $90 \%$ by 1 year of cochlear implant use. Although all patients underwent a trial with hearing aids, only three had used acoustic amplification prior to cochlear implantation with some improvement of hearing sensitivity but no advantage in speech perception. 
The failure of acoustic amplification in non-isolated AN can be explained by the mechanisms underlying the hearing disorder. Because in the majority of patients the underlying lesion results in reduction of spike triggering and slowed conduction velocity in residual axons, increasing the intensity of acoustic input can hardly restore synchrony of nerve fiber discharge. In contrast, electrical stimulation through cochlear implants may improve speech perception by bypassing the site of lesion and/or by stimulating spared nerve fibers.

\section{NONUSERS OF ASSISTIVE DEVICES}

Six patients of 34 with isolated $A N$, and four subjects of 14 with non-isolated AN did not use any assistive device (Table 2). The reasons were refusal to undergo cochlear implantation, missing appointments on follow-up, and not being selected for cochlear implantation.

\section{CHILDREN DISCHARGED FROM NICUs}

Over $50 \%$ (13 of 23) of children showing the electrophysiological profile of $\mathrm{AN}$ who are followed up at our institution (Table 2) are hearing aid users, which is in line with the findings reported by Rance and colleagues. ${ }^{32}$ Of the remaining children, four showed severe to profound deafness and underwent cochlear implantation after a trial of hearing aid use, and three proved to have normal hearing thresholds with normal speech perception and obviously are not using any assistive device. Importantly, $\sim 70 \%$ of children had a difficult neonatal course and showed signs of CNS pathology. As a consequence, speech perception abilities could not be evaluated in most of them. In contrast, reliable hearing thresholds have been obtained at different times from all.

On average, hearing aid users showed moderate hearing loss and aided thresholds pointed to good functional gain. Speech perception abilities could be evaluated in four children only, with scores of 80 to $90 \%$ on open-set recognition of disyllabic words.
In conclusion, the majority of children included in this group appear to be good hearing aid users. This is deemed to ensue from the pathophysiological mechanism underlying AN, which should mainly be related to IHC dysfunction.

\section{CONCLUSION}

The outcome of different rehabilitative options in patients with $\mathrm{AN}$ shows a wide range of variability. Some patients are poor users of hearing aids and cochlear implants, others achieve variable improvement in hearing sensitivity with assistive device use, and yet other patients have a definite advantage. This wide variability results from both site and extension of lesion as well as the precise mechanisms disrupting fast secure transmission of auditory information from the cochlea to the brainstem. In general, the majority of patients with genetic AN benefit from cochlear implant use with a clear advantage for those with isolated AN. In contrast, over $50 \%$ of children discharged from NICUs are good hearing aid users. Due to the high incidence of CNS involvement in this population of children with consequent difficulty in the evaluation of rehabilitation outcome, caution should be exercised in the process of selection for cochlear implantation.

\section{REFERENCES}

1. Starr A, Zeng FG, Michalewski HJ, et al. Perspectives on auditory neuropathy: disorders of inner hair cell, auditory nerve, and their synapse. In: The Senses: A Comprehensive Reference. New York, NY: Academic Press; 2008:397-412

2. Zeng F-G, Kong Y-Y, Michalewski HJ, Starr A. Perceptual consequences of disrupted auditory nerve activity. J Neurophysiol 2005;93:3050-3063

3. Starr A, Picton TW, Sininger Y, Hood LJ, Berlin CI. Auditory neuropathy. Brain 1996;119:741-753

4. Starr A, Sininger YS, Pratt $H$. The varieties of auditory neuropathy. J Basic Clin Physiol Pharmacol 2000;11:215-230

5. Santarelli R. Information from cochlear potentials and genetic mutations helps localize the lesion site in auditory neuropathy. Genome Med 2010;2:91

6. Johnson JS, Newport EL. Critical period effects on universal properties of language: the status of subjacency in the acquisition of a second language. Cognition 1991;39:215-258 
7. Butinar D, Starr A, Zidar J, Koutsou P, Christodoulou K. Auditory nerve is affected in one of two different point mutations of the neurofilament light gene. Clin Neurophysiol 2008;119:367-375

8. Santarelli R, Starr A, Michalewski HJ, Arslan E. Neural and receptor cochlear potentials obtained by transtympanic electrocochleography in auditory neuropathy. Clin Neurophysiol 2008;119: 1028-1041

9. Pangrsic T, Lasarow L, Reuter K, et al. Hearing requires otoferlin-dependent efficient replenishment of synaptic vesicles in hair cells. Nat Neurosci 2010;13:869-876

10. Roux I, Safieddine S, Nouvian R, et al. Otoferlin, defective in a human deafness form, is essential for exocytosis at the auditory ribbon synapse. Cell 2006;127:277-289

11. Santarelli R, Del Castillo I, Rodríguez-Ballesteros $\mathrm{M}$, et al. Abnormal cochlear potentials from deaf patients with mutations in the otoferlin gene. J Assoc Res Otolaryngol 2009;10:545-556

12. Starr A, Michalewski HJ, Zeng F-G, et al. Pathology and physiology of auditory neuropathy with a novel mutation in the MPZ gene (Tyr145- > Ser). Brain 2003;126:1604-1619

13. Huang T, Santarelli R, Starr A. Mutation of OPA1 gene causes deafness by affecting function of auditory nerve terminals. Brain Res 2009;1300:97-104

14. Williams PA, Morgan JE, Votruba M. Opa1 deficiency in a mouse model of dominant optic atrophy leads to retinal ganglion cell dendropathy. Brain 2010;133:2942-2951

15. Kjer P, Jensen OA, Klinken L. Histopathology of eye, optic nerve and brain in a case of dominant optic atrophy. Acta Ophthalmol (Copenh) 1983; 61:300-312

16. Alavi MV, Bette $\mathrm{S}$, Schimpf $\mathrm{S}$, et al. A splice site mutation in the murine Opa1 gene features pathology of autosomal dominant optic atrophy. Brain 2007;130:1029-1042

17. American Academy of Pediatrics, Joint Committee on Infant Hearing. Year 2007 position statement: Principles and guidelines for early hearing detection and intervention programs. Pediatrics 2007; 120:898-921

18. Xoinis K, Weirather Y, Mavoori H, Shaha SH, Iwamoto LM. Extremely low birth weight infants are at high risk for auditory neuropathy. J Perinatol 2007;27:718-723

19. Kraus N, Ozdamar O, Stein L, Reed N. Absent auditory brain stem response: peripheral hearing loss or brain stem dysfunction? Laryngoscope 1984;94:400-406

20. Arslan E, Turrini M, Lupi G, Genovese E, Orzan E. Hearing threshold assessment with auditory brainstem response (ABR) and ElectroCochleoGraphy $(\mathrm{ECoch} \mathrm{G})$ in uncooperative children. Scand Audiol Suppl 1997;46:32-37
21. Jiang ZD, Brosi DM, Shao XM, Wilkinson AR. Sustained depression of brainstem auditory electrophysiology during the first months in term infants after perinatal asphyxia. Clin Neurophysiol 2008; 119:1496-1505

22. Jiang ZD, Wu YY, Liu XY, Wilkinson AR. Depressed brainstem auditory function in children with cerebral palsy. J Child Neurol 2011;26: 272-278

23. Amatuzzi M, Liberman MC, Northrop C. Selective inner hair cell loss in prematurity: a temporal bone study of infants from a neonatal intensive care unit. J Assoc Res Otolaryngol 2011;12:595-604

24. Roush P, Frymark T, Venediktov R, Wang B. Audiologic management of auditory neuropathy spectrum disorder in children: a systematic review of the literature. Am J Audiol 2011;20: 159-170

25. Rance G, Barker EJ. Speech and language outcomes in children with auditory neuropathy/dyssynchrony managed with either cochlear implants or hearing aids. Int J Audiol 2009;48:313-320

26. Teagle HFB, Roush PA, Woodard JS, et al. Cochlear implantation in children with auditory neuropathy spectrum disorder. Ear Hear 2010;31: 325-335

27. Rodríguez-Ballesteros M, del Castillo FJ, Martín $\mathrm{Y}$, et al. Auditory neuropathy in patients carrying mutations in the otoferlin gene $(O T O F)$. Hum Mutat 2003;22:451-456

28. Miyamoto RT, Kirk KI, Renshaw J, Hussain D. Cochlear implantation in auditory neuropathy. Laryngoscope 1999;109:181-185

29. Brookes JT, Kanis AB, Tan LY, Tranebjaerg L, Vore A, Smith RJ. Cochlear implantation in deafness-dystonia-optic neuronopathy (DDON) syndrome. Int J Pediatr Otorhinolaryngol 2008;72: 121-126

30. Starr A, Isaacson B, Michalewski HJ, et al. A dominantly inherited progressive deafness affecting distal auditory nerve and hair cells. J Assoc Res Otolaryngol 2004;5:411-426

31. Breneman AI, Gifford RH, Dejong MD. Cochlear implantation in children with auditory neuropathy spectrum disorder: long-term outcomes. J Am Acad Audiol 2012;23:5-17

32. Rance G, Cone-Wesson B, Wunderlich J, Dowell R. Speech perception and cortical event related potentials in children with auditory neuropathy. Ear Hear 2002;23:239-253

33. Lee DS, Lee JS, Oh SH, et al. Cross-modal plasticity and cochlear implants. Nature 2001; 409:149-150

34. Boothroyd A. The acoustic speech signal. In: Pediatric Audiology: Diagnosis, Technology, and Management. New York, NY: Thieme; 2008: 159-167 
35. Varga R, Kelley PM, Keats BJ, et al. Non-syndromic recessive auditory neuropathy is the result of mutations in the otoferlin (OTOF) gene. J Med Genet 2003;40:45-50

36. Delmaghani S, del Castillo FJ, Michel V, et al. Mutations in the gene encoding pejvakin, a newly identified protein of the afferent auditory pathway, cause DFNB59 auditory neuropathy. Nat Genet 2006;38:770-778

37. Schoen CJ, Emery SB, Thorne MC, et al. Increased activity of Diaphanous homolog 3 (DIAPH3)/ diaphanous causes hearing defects in humans with auditory neuropathy and in Drosophila. Proc Nat. Acad Sci USA 2010;107:13396-13401

38. Wang Q, Li R, Zhao H, et al. Clinical and molecular characterization of a Chinese patient with auditory neuropathy associated with mitochondrial 12S rRNA T1095C mutation. Am J Med Genet A 2005;133A:27-30

39. Kovach MJ, Campbell KCM, Herman K, et al. Anticipation in a unique family with CharcotMarie-Tooth syndrome and deafness: delineation of the clinical features and review of the literature. Am J Med Genet 2002;108:295-303

40. Kalaydjieva L, Gresham D, Gooding R, et al. $\mathrm{N}$-myc downstream-regulated gene 1 is mutated in hereditary motor and sensory neuropathy-Lom. Am J Hum Genet 2000;67:47-58

41. López-Bigas N, Olivé M, Rabionet R, et al. Connexin 31 (GJB3) is expressed in the peripheral and auditory nerves and causes neuropathy and hearing impairment. Hum Mol Genet 2001;10:947-952

42. Bähr M, Andres F, Timmerman V, Nelis ME, Van Broeckhoven C, Dichgans J. Central visual, acoustic, and motor pathway involvement in a CharcotMarie-Tooth family with an Asn205Ser mutation in the connexin 32 gene. J Neurol Neurosurg Psychiatr 1999;66:202-206

43. Amati-Bonneau P, Guichet A, Olichon A, et al. OPA1 R445H mutation in optic atrophy associated with sensorineural deafness. Ann Neurol 2005; 58:958-963

44. Meyer E, Michaelides M, Tee LJ, et al. Nonsense mutation in TMEM126A causing autosomal recessive optic atrophy and auditory neuropathy. $\mathrm{Mol}$ Vis 2010;16:650-664

45. Rance G, Fava R, Baldock H, et al. Speech perception ability in individuals with Friedreich ataxia. Brain 2008;131:2002-2012

46. Wang QI, Li QZ, Rao SQ, et al. AUNX1, a novel locus responsible for $\mathrm{X}$ linked recessive auditory and peripheral neuropathy, maps to Xq23-27.3. J Med Genet 2006;43:e33

47. Bahmad F, Merchant SN, Nadol JB, Tranebjaerg L. Otopathology in Mohr-Tranebjaerg syndrome. Laryngoscope 2007;117:1202-1208

48. Ceranić B, Luxon LM. Progressive auditory neuropathy in patients with Leber's hereditary optic neuropathy. J Neurol Neurosurg Psychiatr 2004;75: 626-630 